\title{
Ethnobotanical plants of Veligonda Hills, Southern Eastern Ghats, Andhra Pradesh, India
}

\author{
S K M Basha ${ }^{1 *}$ and P Siva Kumar Reddy ${ }^{2}$ \\ ${ }^{1}$ NBKR Medicinal Plant Research Institute, Vidya Nagar, SPSR Nellore, Andhra Pradesh, India \\ ${ }^{2}$ Research and Development Centre, Bharathiyar University, Coimbatore, Tamil Nadu, India
}

Article history

Received: 04 September 2016

Accepted: 16 October 2016

Published: 01 January 2017

(C) Basha \& Siva Kumar Reddy (2017)

Editor

K. K. Sabu

Publisher

Horizon e-Publishing Group

Corresponding Author

S K M Basha

区drskmbasha@gmail.com

\begin{abstract}
The Veligonda range which separates the Nellore district from Kadapa and Kurnool is the back bone of the Eastern Ghats, starting from Nagari promontory in Chittoor district. It runs in a northerly direction along the western boarders of the Nellore district, raising elevation of 3,626 feet at Penchalakona in Rapur thaluk. Veligonda hill ranges have high alttudinal and deep valley. These hills have rich biodiversity and many rare, endangered, endemic and threatned plants are habituated in these hills. The present paper mainly deals with the ethanobotanical plants used by local people.
\end{abstract}

\section{Keywords}

Ethnobotany; Threatened; Endangered; Endemic; Veligonda hill range

Basha, S. K. M., and P. Siva Kumar Reddy. 2017. Ethnobotanical plants of Veligonda Hills, Southern Eastern Ghats, Andhra Pradesh, India. Plant Science Today 4(1): 1-11. http://dx.doi.org/10.14719/pst.2017.4.1.268

\section{Introduction}

The World Health Organization (WHO) estimated that $80 \%$ of the population of developing countries relies on traditional medicines, mostly plant drugs, for their primary healthcare needs. Also, modern pharmacopoeia still contains at least 25\% drugs derived from plants and many others which are synthetic analogues built on prototype compounds isolated from plants. Demand for medicinal plant is increasing in both developing and developed countries due to growing recognition of natural products, being non-narcotic, having no side-effects, easily available at affordable prices and sometime the only source of health care available to the poor.

It is evident that the Indian people have tremendous passion for medicinal plants and use them for wide range of health related applications from a common cold to memory improvement and treatment of poisonous snake bites to a cure for muscular dystrophy and the enhancement of body's general immunity. In the oral traditions, local communities in every ecosystem from the Trans Himalayas down to the coastal plains have discovered the medical uses of thousands of plants found locally in their ecosystem. India has one of the richest plant medical cultures in the world. It is a culture that is of tremendous contemporary relevance because it can on one hand ensure health security to millions of people and on the other hand it can provide new and safe herbal drugs to the entire world. There are estimated to be around 25000 effective plant based formulations used in folk medicine and known to rural communities all over India and around 10000 designed formulations are available in the indigenous medical texts.

\section{Study area}

Eastern Ghats are one of the nine major floristic zones of India possessing rich and diversified plant wealth due to undulated topography and availability of rich humus content. The forests of Eastern Ghats 
in Andhra Pradesh are inhabited with 33 tribal 9 groups (Tribal Welfare Department, Government of Andhra Pradesh, 2011). They contain valuable information regarding therapeutic properties of commonly used crude drugs for different human and veterinary ailments which was recorded and critically analyzed with the help of literature as well as field observations. Based on these observations some potential drug yielding plants, which have limited distribution, were selected for scientific evaluation. Most of the enumerated taxa were reported as endemic and endangered (Nair and Sastry, 1998) as they have been over-exploited for different purposes.

\section{Eastern Ghats, an overview}

The Eastern Ghats cover on area about 75,000 sq. $\mathrm{km}$. traversing the coromandel between $11^{\circ} 30^{\prime}$ $22^{\circ} \mathrm{N}$ latitudes and $76^{\circ} 50^{\prime}-86^{\circ} 30^{\prime} \mathrm{E}$ longitudes. Its northern boundary is marked by river Mahanadi basin while the southern boundary is the Cauvery and west lives tips Bastar, Telangana, Karnataka plateaus and Tamil Nadu uplands. They pass mainly in three states viz. Orissa, Andhra Pradesh and Tamil Nadu.

In Andhra Pradesh, Eastern Ghats pass through Srikakulam, East Godavari, West Godavari, Khammam, Krishna, Guntur, Mahaboobnagar, Prakasam, Kurnool, Kadapa, Nellore and Chittoor districts. Eastern Ghats do not form continuous range like Western Ghats but assemblage of discontinuous ranges of hills with plateaus, escarpments, butters, tors, narrow basins and gorges with elevation ranging from few meters to more than $1600 \mathrm{~m}$. The Mahanadi, the Godavari, the Pennar and the Cauvery are main rivers which raise in Western Ghats have cut extensively through Eastern Ghats to escape into the Bay of Bengal, hence, they do not form a continuous range. Based on the climates, topographic, geographical features the Eastern Ghats of Andhra Pradesh can be divided into the following regions:

1. Northern Eastern Ghats: The stretching extreme north of the state i.e. Simhachalam and Rampa hills.

2. Southern Eastern Ghats: These Ghats stretching between the South of the river, the cannery through Papi hills, Kondapalli range, Nallamalais, Yerramalais, Palakonda, Veligonda range, Horseley hills, Seshachalam hills, Nagari hills, etc.

The altitudes in the Eastern Ghats of Andhra Pradesh range from $300-1500 \mathrm{~m}$ above MSL. The altitudes more than $1000 \mathrm{~m}$ above MSL in central parts of the north Eastern Ghats and $300-600 \mathrm{~m}$, and above in Southern Eastern Ghats, while in Nallamalais the highest peak in rising between 600 $-800 \mathrm{~m}$ above MSL.

\section{Veligonda Hills}

Veligonda and adjoining hill ranges spread along about $170 \mathrm{~km}$ North to South in Kadapa and Nellore Districts and stretching a little further into Prakasam district. Geographically these hill ranges lie between $79^{\circ} \mathrm{E}$ to $79^{\circ} 30^{\prime} \mathrm{E}$ and $13^{\circ} 45^{\prime} \mathrm{N}$ to $15^{\circ} 15^{\prime}$ $\mathrm{N}$. The latitude in general ranges up to $1000 \mathrm{~m}$. The forests are in general dry deciduous type. Veligonda and adjoining hill ranges comprising of Palakonda, Seshachalam, Lankamala and terminal part of Nallamalais from mid region of Southern Eastern hats.

The Veligonda range which separates the Nellore district from Kadapa and Kurnool is the backbone of the Eastern Ghats, starting from Nagari promontory in Chittoor district. It runs in a northerly direction along the western boarders of the Nellore district, raising elevation of $1105 \mathrm{~m}$ at Penchalakona in Rapur thaluk. Veligonda hill ranges have high altitudinal and deep valleys. Among the Velugondas range of hills the Durgam in Venkatagiri range and Penchalakona are the most prominent and $914 \mathrm{~m}$ above msl.

\section{Vegetation types in Eastern Ghats in Andhra Pradesh}

The forest area of the State extends about 63,814 sq $\mathrm{km}$ which constitute $23.2 \%$ of the total land area (Forest Report of Andhra Pradesh, 2013). The vegetation in Eastern Ghats is determined by climate, edaphic factors and biotic factors along with altitude. The wide variations in climate and topography of the Eastern Ghats have resulted in various types of forest growth. The vegetation in Eastern Ghats is classified based on the concept of Champion and Seth (1968). The following types of vegetation are found in Eastern Ghats of Andhra Pradesh.

\section{Tropical moist deciduous vegetation}

These are typical deciduous forest with high annual rainfall of over $1000 \mathrm{~mm}$ and mixed with evergreen species only along the patches of selected habitats. This type of forests found in Eastern Ghats of Andhra Pradesh like, Ananthagiri, Maredumilli, and East Godavari District. It has the following prominent sub types viz., a) Forests dominated with sal (Shorea robusta Roth., Dipterocarpaceae), b) Those completely devoid of sal or Non sal forests, and c) Rivarian forests found along with river banks, streams and in the low hilly areas.

The vegetation is characterized by dense foliage at top canopy with abundant large climbers and epiphytes with scattered bamboo growth. The deciduous period is very less i.e., March-April. These can be sub classified into following categories.

\section{a) Northern Tropical moist deciduous sal forests}

This type of forest sub type found at Donubai area, Srikakulam, Vijayanagaram and Seshachalam hills 
of Chittoor and Kadapa Districts. The most dominant tree species are Shorea robusta Roth. (Dipterocarpaceae) along with other co dominate species like Xylia xylocarpa (Roxb.) Taub (Fabaceae), Haldinia cordifolia Roxb. (Rubiaeae), Anogeissus latifolia (Roxb ex. DC.) Wall. ex Guillem. \& Perr (Combretaceae), Terminalia alata Roth. (Combretaceae), Lannea coromandelica (Houtt.) Merr (Anacardiaceae), Madhuca longifolia (Koen.) Macbr. (Sapotaceae), Albizzia procera (Roxb.) Benth (Fabaceae), Syzygium cumini (L.) Skeels (Myrtaceae), Pterocarpus marsupium Roxb. (Fabaceae) mixed with species of middle canopy like Cleistanthus collinus (Roxb. Benth. ex. Hook.f), Dillinia pentagyna Roxb. (Dillineaceae). The lower canopy with shrubby species Alstonia venenata R.Br. (Apocynaceae), Cipadessa baccifera (Roth.) Miq. (Meliaceae), Woodfordia fruiticosa (L.) Kurz (Lythraceae) Helictres isora L. (Sterculiaceae), etc. and ground is covered with some herbs, like Desmodium pulchellum (L.) Benth. (Fabaceae), Curcuma neilghierensis Wight. (Zingebereace), Globba merantina L. (Zingeberaceae), Tephrosia tinctorea Pers. (Fabaceae), etc. along with grass species like Arundinella setacea Trin. (Poaceae), Apluda muitica L. (Poaceae), etc. A few evergreen species like, Syzygium cumini L. (Myrtaceae), Memecelon umbellatum Burm.f. (Lythraceae), Diospyros malabarica (Desr.) Kostel (Ebenaceae), D. melanoxylon Roxb. (Ebenaceae), etc.

\section{b) Southern Tropical moist deciduous (Non-Sal) forests}

These are found in the districts of Vizayanagaram (Punyagiri area), Visakhapatnam (Gudem), East Godavari (Rampa), West Godavari (Polavaram), Kurnool (Nallamalais), which contain the dominant species like Anogeissus latifolia (Roxb ex. DC.) Wall. ex Guillem. \& Perr (Combretaceae), Dalbergia latifolia, Roxb. (Fabaceae) Mangifera indica L. (Anacardiaceae), Pterocarpus marsupium Roxb. (Fabaceae), Terminalia alata Roth. (Combretaceae), Sterculea urens Roxb. (Sterculiaceae) and Xylia xylocarpa (Roxb.) Taub (Fabaceae) represents top canopy mixed with middle canopy species like Bridelia retusa (L.) A. Juss, Careya arborea Roxb (Lycythidaceae), Grewia tilifolia Vahl. (Tiliaceae), Glochidion zeylanicum (Gaertn.) Juss. (Euphorbiaceae), Holarrhena pubescens (BuchHam) Wall. ex. Don (Apocynaceae), Litsea glutinosa (Lour.) C. B. Rob. (Lauraceae), Mallotus philippensis (Lam.) Mull. Arg. (Euphorbiaceae), Polyalthea cerasoides (Roxb.) Bedd. (Annonaceae) along with bamboo breaks commonly of Dendrocalamus strictus (Roxb.) Ness (Poaceae) and occasionally of Bambusa arundinacia (Retz.) Willd. (Bamboosaceae) and teak also found in some plantations. The middle and lower canopy with the species of Cissus vitigenea L. (Vitaceae), Gardenia gummifera L. f. (Rubiaceae), Helectres isora L. (Sterculiaceae), Ixora arborea Roxb. ex. Smith (Rubiaceae), Nyctanthus arbor-tristis L. (Oleaceae), Woodfordia fruiticosa (L.)
Kurz (Lythraceae), etc. The low shrubby layer mixed with tall grasses such as Apluda mutica L. (Poaceae), Themeda triandra Forssk. (Poaceae), Chlorophytum tuberosum (Roxb.) Baker (Liliaceae), Pimpinella tirupatiensis N. P. Balakr. \& Subram. (Apiaceae), etc. along with lianas of Bauhinia vahlii Wight \& Arn., Entada pursaetha DC. (Mimosaceae), Toddalia asiatica Lam. (Rutaceae), etc with ground species.

\section{c) Southern Tropical moist deciduous riparian forest}

These are common along with river banks (Godavari), with semi evergreen species like Barringtonia acutangula (L.) Gaertn. (Baringtoniaceae), Ficus racemosa L. (Moraceae), Homonia riparia Lour. (Euphorbiaceae), Terminalia arjuna (Roxb. \& DC.) Wight \& Arn.(Combretaceae), Mimosa pudica L. (Mimosaceae), Syzygium cumini (L.) Skeels (Myrtaceae), etc. The dominant grass in this vegetation type is Saccharum spontaneum L. (Poaceae).

\section{Tropical dry deciduous forests}

This represents typical deciduous forest growing in larger areas along with the northern, middle and southern Eastern Ghats. The upper canopy in these forests are uneven with mixture of species mostly typical deciduous trees which become leafless during dry seasons. Shrubs and grasses grow as undergrowth in a limited density in frequent forest fires. Bamboo and woody climbers being exposed, moist areas along low stream banks are the suitable habitats for epiphytes. These forests are classified into following sub types:

\section{a) Teak - bearing dry deciduous forests}

These are distributed mostly in Eastern Ghats of Visakhapatnam, East Godavari, West Godavari, Khammam districts and Rayalaseema region. The teak is associated with Anogeissus latifolia (Roxb ex. DC.) Wall. ex Guillem. \& Perr (Combretaceae), Boswellia serrata Roxb. ex. Colebr (Burseraceae), Cassia pinnata, Chloroxylon swietenia DC. (Rutaceae), Garuga pinnata Roxb., Pterocarpus marsupium Roxb. (Fabaceae), Terminalia alata Roth., T. chebula Retz., T. bellirica (Gaertn.) Roxb. (Combretaceae), etc., mixed with shrub species like Canthium dicoccum (Gaertn.) Merr (Rubiaceae), Chomelia asiatica (L.) Kunze. (Rubiaceae), Erythroxylon monogynum Roxb. (Erythroxylaceae), Holarrhena pubescens Wall. ex. G. Don (Apocynaceae), Helecteris isora L. (Sterculiaceae), etc., covered by climbers like Bauhinia vahli Wight \& Arn. (Caesalpiniaceae), Cissus pallida (Wight \& Arn.) Planchon (Vitaceae), Mucuna pruriens (L.) DC. (Fabaceae), Ventilago maderaspatana Gaertn (Rhamnaceae). The ground layer is covered gregariously with bamboo bushes, Dendrocalamus strictus (Roxb.) Nees (Poaceae) and other tall 
grasses like Curcuma pseudomontana Graham. (Zingiberaceae), etc.

\section{b) Non - Teak dry deciduous forests}

These forests are found in the districts of Rayalaseema and Nellore, interestingly some parts of these forests are dominated by endemic species like Pterocarpus santalinus L. f. (Fabaceae), Shorea tumbaggaia Roxb. (Dipterocarpaceae) on the hill tops of Seshachalam (Tirumala) hills and Syzygium alternifolium (Wight) Walp. (Myrtaceae) is also sub dominant species in these areas. The other endemics like Boswellia ovalifoliolata N. P. Balakr. \& Henry (Burseraceae), Cycas beddomei Dyer (Cycadaceae), Pimpinella tirupetiensis N. P. Balakr. \& Subram. (Apiaceae), Rhynchosia beddomei Baker (Faabaceae) and Actinodaphne madraspatana Bedd. ex. Hook. $\mathrm{f}$ (Lauraceae) are not uncommon in above area. This can be termed as gaps in Seshachalam hills growing along with some common elements like Terminalia pallida (endemic tree) Brandis (Combretaceae) T. alata Roth. (Combretaceae), Bridelia retusa (L.) A. Juss (Euphorbiaceae), Pinus roxburghii Sarg. (Pinaceae) (exotic) Acacia auriculiformis Benth. (Mimosoideae) (introduced), mixed with dry deciduous elements like Erythroxylum monogynum Roxb. (Erythroxylaceae), Ziziphus maurintiana Lam. (Rhamnaceae), etc. The climbers are Celastrus paniculata Willd. (Celastraceae), The common grasses found in these forests are Cymbopogon coloratus (Hoof. f.) Stapf and Heteropogon contortus. (L.) P. Beauv. ex Roem. \& Schult. (Poaceae) On the hill tops gregarious patches of Phoenix loureirii Kunth (Aracaceae) are also found.

\section{Mixed dry deciduous forests}

These forests are found in drier localities in Rayalaseema region (Anantapuram, Chittoor, Kadapas and Kurnool) of Eastern Ghats. In these forests a mixed type of Vegetation is seen. These forests are classified into following sub types:

\section{a) Southern - mixed dry deciduous forests}

These are more common in drier localities and subjected to extreme biotic interference like grazing, fires and collection centers of NWFP, found mostly in all districts of Rayalaseema region of Eastern Ghats. The floristic components comprises Gardenia gummifera L. f. (Rubiaceae), different species of Terminalia, Albizia and Acacias, Pterocapus marsupium Roxb. (Fabaceae), Hardwickia binata Roxb. (Fabaceae), Balanites eagiptica (L.) Del (Balantiaceae), etc. along with gregarious growth of Phoenix loureirii Kunth. (Arecaceae) and Chloroxylon swietenia DC. (Rutaceae). The hill slopes are found with Boswellia serrata Roxb. ex. Colebr., Commiphora caudate (Wight \& Arn.) Engl. (Burseraceae), Terminalia arjuna Roxb. ex DC.) Wight \& Arn.(Combretaceae) (trenches of hill slopes). The climbers like Decalepis hamiltonii Wight \& Arn (Asclepiadaceae) Gymnema sylvestre (Retz.) R. Br. ex Sm. (Asclepiadaceae), etc., found in open areas of Nallamalais, Seshachalam and Yerramalais of the Eastern Ghats.

\section{b) Northern mixed dry deciduous forests}

These forests are not frequent but present in northern and southern corners like Orissa and Tamil Nadu states respectively.

\section{Dry evergreen forests}

This type of forests occur in coastal plains like Vijayanagaram (Poolbagh), Visakhapatnam (Madugula), Srikakulam (Pathapatnam), Nellore (Sriharikota) and Chittoor (Mamandur) district. Sriharikota Island is located in Nellore district of southern part adjoining the Pulicat Lake. This island and its surroundings support dry evergreen vegetation. The common species found in these forests are Albizia amara, Manilkara hexandra, Sapindus emarginatus and Strychnos nux-vomica. The climbers like Strychnos minor, Pyrenacanthus volubilis, and Derris scandens.

\section{Thorny - Scrub forests}

These are degraded deciduous forests due to biotic interference, over exploitation, and frequent fires and are widely distributed in arid and semiarid parts of Eastern Ghats especially in forest peripheries. Hence the climax was changed to thorny scrub forests (secondary in origin). Due to frequent forest fires some fire resistant spiny species like Lantana camara L. (Verbanaceae), Ziyziphus oenoplea (L.) Mill (Rhamnaceae) established as invaders. In some places Hyptis suaveolens (L.) Poit (Lamiaceae) and Cassia alata L. (Caesalpiniaceae) are also found as invaders. These forests supports the growth of grass species for short period of rainy season and vegetation termed as dry Savannah forests (*In fact Savannah are native to African countries). Typical species are Chloroxylon swietenea DC. (Rutaceae), Terminalia alata Roth. (Combretaceae), Atalantia monophylla (Roxb.) DC (Rutaceae), Capparis zeylanica L. (Capparaceae), Cadaba fruiticosa (L.) Druce (Capparaceae), Zyziphus Mauritania Lam (Rhamnaceae)., Z. xylopyrus (Retz.) Willd (Rhamnaceae), Euphorbia antiquorum L., E. tirucalli L. (Euphorbiaceae), Flacourtia indica (Burm. f.) Merr (Flacourtiaceae), Dodonaea viscose (L.) Jacq. (Sapindaceae), Cassia auriculata (L.) Roxb. (Caesalpiniaceae), Dichrostachys cineria (L.) Wight \& Arn (Mimosaceae), etc. with stunted growth bearing elements of Terminalia alata Roth (Combretaceae), Anogeissus latifolia (Roxb ex. DC.) Wall. ex Guillem. \& Perr (Combretaceae), Pterocarpus marsupium Roxb. (Fabaceae), etc. The grass species like Apluda mutica L. (Poaceae), Themeda triandra Forssk (Poaceae), Cymbopogon sp. etc., are also common. 
Table 1. Plants of Veligonda hills

\begin{tabular}{|c|c|c|c|c|c|}
\hline S. No & Botanical name & Vernacular name & Family & Habit & Medicinal uses \\
\hline 1 & Abrus precatorius L. & Gurivinda & Fabaceae & Climber & Anti inflamatery \\
\hline 2 & Abuliton indicum (L.) Sweet. & $\begin{array}{l}\text { Duvvenakaya/ } \\
\text { Tutturubenda }\end{array}$ & Malvaceae & Shrub & Haematuria \\
\hline 3 & Acacia leucophloea (Roxb.) Willd. & Tella tumma & Mimosaceae & Tree & Diuretic \\
\hline 4 & Acacia tora (Roxb.) Craib. & Korinteega & Mimosaceae & Climber & \\
\hline 5 & Achyranthes aspera L. & Uttareni & Amaranthaceae & Herb & Diuretic; Piles \\
\hline 6 & Actinopteris radiate (Sw.) Link. & Mayuri shika & Actinopteridaceae & Herb & Skin diseases \\
\hline 7 & Adiantum caudatum $\mathrm{L}$. & Raja hamsa & Adiantaceae & Herb & Diabetes \\
\hline 8 & Aegle marmelos (L.) & Maredu / Bilva & Rutaceae & Shrub & Dysentery \\
\hline 9 & Aerva lanata (L.) Juss. & Pindikura & Amaranthaceae & Herb & Urinary diseases \\
\hline 10 & Ageratum conyzoides $\mathrm{L}$. & Goat weed & Asteraceae & Herb & Nervine tonic \\
\hline 11 & $\begin{array}{l}\text { Alangium salvifollium (L.f.) } \\
\text { Wangerin }\end{array}$ & Udaga / Ankolamu & Alangiaceae & Tree & Dog Bite \\
\hline 12 & Albizia amara (Roxb.) B.Boivin & Cheekireni & Mimosaceae & Tree & Inflamation \\
\hline 13 & Albizzia odoratissima (L.f.) Benth & Chinduga & Mimosaceae & Tree & Leprosy \\
\hline 14 & Alstonia scholaris L. & Edakulapala & Apocynaceae & Climber & Galactagogue,asthma \\
\hline 15 & $\begin{array}{l}\text { Andrographis paniculata (Burm.f.) } \\
\text { Wall. }\end{array}$ & Nelavemu & Acanthaceae & Herb & Fever \\
\hline 16 & Anisomelea malabarica (L.) & Moga-Bira & Lamiaceae & Shrub & Eczema; Diarrhoea \\
\hline 17 & Annona squamosa L. & Sitapalem & Annonaceae & Tree & Abortifacient \\
\hline 18 & Annona reticulate $\mathrm{L}$. & Ramapalam & Annonaceae & Tree & Astringent \\
\hline 19 & $\begin{array}{l}\text { Anogeissus latifolia (Roxb.ex Dc.) } \\
\text { Wall.ex Guillem.\&Perr }\end{array}$ & Chirimanu / Elama & Combretaceae & Tree & Piles; Snake bite \\
\hline 20 & Argemeone mexicana L. & $\begin{array}{l}\text { Kusuma / } \\
\text { Brahmadandi }\end{array}$ & Pepepaveraceae & Herb & Syphilis \\
\hline 21 & Aristolochia braceteolata Lam. & Gadidagadapa & Aristolochiaceae & Herb & Eczema; Leprosy \\
\hline 22 & Aristolochia indica L. & Easwari & Aristolochiaceae & Herb & Snake bite \\
\hline 23 & Asperagus racemosus Willd. & Sathavari & Liliaceae & Herb & Diabetes; Leucorrhoea \\
\hline 24 & Atalantia monophylla (L.) & Munukudu & Rutaceae & Shrub & Antiseptic; Fever \\
\hline 25 & Atylosia scarabaeoides (L.) Benth. & Adavikandi & Fabaceae & Climber & \\
\hline 26 & Azadirachta indica A.Juss. & Vepa & Meliaceae & Tree & Skin diseases \\
\hline 27 & Azima tetracantha Lam. & Tella uppili & Salvadoraceae & Shrub & Leprosy; Eczema \\
\hline 28 & Bacopa monnieri (L.) Pennel & Brahmi & Scrophulriaceae & Herb & Memory booster \\
\hline 29 & Basella alba L. & Bachali & Basellaceae & Climber & Constipation \\
\hline 30 & Bauhinia racemosa Lam. & Are fibres & Caesalpinaceae & Tree & Malaria Fever \\
\hline 31 & Blumea mollis (D.Don) Merr. & Kukkapogaku & Asteraceae & $\begin{array}{l}\text { Aromatic } \\
\text { erect herb }\end{array}$ & Dropsy; Throat infection \\
\hline 32 & Boerhavia diffusa $\mathrm{L}$. & Attamamidi & Nyctaginaceae & Herb & Urinary disorders \\
\hline 33 & Bombax ceiba $\mathrm{L}$. & Adavi Buruga & Malvaceae & Tree & Diabetes; Diuretic \\
\hline 34 & Borassus flabellifer L. & Tati & Araceae & Tree & Odema; Constipation \\
\hline 35 & Boswelia ovalifoliata Bal. \& Henry & Sambrrani & Burseraceae & Tree & Stomach ulcers; Dysentery \\
\hline 36 & Boswelia serrata Roxb. & Sambrani & Burseraceae & Tree & Arthrites \\
\hline 37 & $\begin{array}{l}\text { Buchnania axilaris (Desr.) } \\
\text { Ramamoorthy }\end{array}$ & Sara & Anacardiaceae & Tree & $\begin{array}{l}\text { Boils; Cardio tonic; } \\
\text { Wounds }\end{array}$ \\
\hline 38 & Butea monosperma (Lam) Taub. & Moduga & Fabaceae & Tree & Jaundice; Astringent \\
\hline 39 & Caesalpinia bonduc (L.) Roxb. & Gacha & Fabaceae & Shrub & Leucorrhoea; Hydrocele \\
\hline 40 & Calophyllum inophyllum L. & Ponna & Calophyllaceae & Tree & Rheumatism; Astringent \\
\hline 41 & Capparis sepiaria $\mathrm{L}$. & Nalla uppili & Capparaceae & Shrub & Febrifuge \\
\hline 42 & Capparis zeylanica $\mathrm{L}$. & Adonda & Capparaceae & Shrub & Antihelmenthic; Sadative \\
\hline 43 & Carissa carundus L. & Vaka & Apocynaceae & Small Tree & Diabetes; Stomachic \\
\hline
\end{tabular}


Table 1. Plants of Veligonda hills - contd.

\begin{tabular}{|c|c|c|c|c|c|}
\hline S. No & Botanical name & Vernacular name & Family & Habit & Medicinal uses \\
\hline 44 & Cardiospermum halicacabum $L$ & Buddakakara & Sapindaceae & Climber & $\begin{array}{l}\text { Rheumatism, Nervous } \\
\text { disorders }\end{array}$ \\
\hline 45 & Careya arborea Roxb. & Budda darimi & Barringtoniaceae & Tree & Eye diseases; Skin sores \\
\hline 46 & Carmona retusa (Vahl) Masam. & $\begin{array}{l}\text { Nomuchettu / } \\
\text { Barranki }\end{array}$ & Boraginaceae & Shrub & Snake bite; Skin diseases \\
\hline 47 & Cassia absus L. & Chanupala vittulu & Caesalpinaceae & Herb & Constipation; Cough \\
\hline 48 & Cassia fistula $\mathrm{L}$. & Rela & Caesalpinaceae & Tree & Diabetes \\
\hline 49 & Cassia italica (Mill.) Spreng. & Nelatangedu & Caesalpinaceae & Herb & Bone fracture \\
\hline 50 & Cassia montana Meyne ex.Roth. & Pyditangedu & Caesalpinaceae & shrub & Body Pains \\
\hline 51 & Cassia occidentalis L.Sp. & Kasintha & Caesalpinaceae & shrub & Laxative \\
\hline 52 & Cassine glauca (Rottb.) Kuntz. & Nerdhi & Celastraceae & & Snake bite; dysuria \\
\hline 53 & Cassytha filiformis $\mathrm{L}$. & Sitamma savaralu & Lauraceae & Climber & Dysentery; Hair tonic \\
\hline 54 & Catunaregam spinosa (Thunb.) Tirv. & Manga & Rubiaceae & Shrub & Diarrohoea; Astringent \\
\hline 55 & Cayratia pedata (Lam.) Gagnep & $\begin{array}{l}\text { Adavi } \\
\text { gummaditeega }\end{array}$ & Vitaceae & Climber & Astringent; Boils \\
\hline 56 & Centella asiatica (L.) Urban. & Saraswathi & Apiaceae & Herb & Brain Tonic \\
\hline 57 & Chloroxylon swietenia DC. & Billudu & Meliaceae & Tree & Rheumatism; Astringent \\
\hline 58 & Cipadessa baccefera (Roth) Miq. & Ranaberi & Meliaceae & Shrub & Diabetes; Wounds \\
\hline 59 & Cissampelos pareira $\mathrm{L}$ & Visha boddi & Menispermaceae & Shrub & Dropsy; Diabetes \\
\hline 60 & Cissus quadrangularis $\mathrm{L}$. & Nalleru & Vitaceae & Herb & Leucorrhoea; Piles \\
\hline 61 & Cissus vetigenia $\mathrm{L}$. & Adavi gummidi & Vitaceae & Climber & Wounds \\
\hline 62 & Citrullus colocynthis (L.) Scharder & Papara & Cucurbitaceae & Climber & Rheumatism; Jaundice \\
\hline 63 & Cocculus hirsutus (L.) Diels & Dusari Teega & Menispermaceae & Climber & Rheumatism \\
\hline 64 & Coccinia grandis J.Voigt. & Kakidonda & Cucurbitaceae & Climber & Diabetes \\
\hline 65 & $\begin{array}{l}\text { Cochlospermum religiosum (L.) } \\
\text { Alston }\end{array}$ & Konda gogu & Cochlospermaceae & Tree & Dysentery; Gonorrhoea \\
\hline 66 & Coldenia procumbens $\mathrm{L}$. & Hamsapadu & Boraginaceae & Herb & Rheumatism \\
\hline 67 & $\begin{array}{l}\text { Commifera caudate (White \&Arn.) } \\
\text { Engl. }\end{array}$ & Kondamamidi & Burseraceae & Small Tree & Rheumatism \\
\hline 68 & $\begin{array}{l}\text { Corallocarpus epigaeus (Rottl.) } \\
\text { C.B.Clark }\end{array}$ & Mukkudonda & Cucurbitaceae & Climber & Eczema; Dysentery \\
\hline 69 & Cordia dichotoma Forst.f & $\begin{array}{l}\text { Bankamanu / } \\
\text { Nakkera }\end{array}$ & Boraginaceae & Tree & Bronchial disorders; Fever \\
\hline 70 & Costus speciosus (Koenig.) Sm. & $\begin{array}{l}\text { Adavi allam/ } \\
\text { Chengalva cost }\end{array}$ & Costaceae & Herb & Dyspepsia; Snake bite \\
\hline 71 & Crateva religiosa G.Forst. & Varuna & Capparaceae & Tree & Kidney stones \\
\hline 72 & Crotalaria retusa $\mathrm{L}$. & Sanapusphi & Fabaceae & Herb & Scabies \\
\hline 73 & Curculigo orchioides Gaertn & Nelathati & Hypoxidaceae & Herb & Diarrhoea; Potency \\
\hline 74 & Cycas beddomi Dyer. & Peritha & Cycadaceae & Tree & Aphrodisiac \\
\hline 75 & Cymopogon flexuosus (L.) Rendle & Nimma gaddi & Poaceae & Herb & Citral oil \\
\hline 76 & Dalbergia latifolia Roxb. & Jittagi / Iridi & Fabaceae & Tree & Ulcers; Leprosy \\
\hline 77 & Dalbergia paniculata Roxb. & Pacchari & Fabaceae & Tree & Filarial Swelling \\
\hline 78 & Datura metal L. & Nalla ummetta & Solanaceae & Herb & Epilepsy \\
\hline 79 & Datura stromonium L. & Ummetta & Solanaceae & Shrub & Asthma; Narcotic \\
\hline 80 & Decalepis hamiltonii Wight \& Arn & Maredu kommulu & Asclepediaceae & Shrub & Haemorrhage; Appetizer \\
\hline 81 & $\begin{array}{l}\text { Decaschistia crotonifolia Wight \& } \\
\text { Arn }\end{array}$ & Adavigogu & Malvaceae & Shrub & Hydrocele \\
\hline 82 & Deccannia pubscens (Roth) Tirveng & Konda manga & Rubiaceae & Tree & Sores \\
\hline 83 & Derris scandens (Roxb.) Benth. & Nalla teega & Fabaceae & Climber & Snake bite \\
\hline 84 & Desmdium triflorum (L.) Dc. & Munta mandu & Fabaceae & Herb & Galactagogue; Diarrhoea \\
\hline
\end{tabular}


Table 1. Plants of Veligonda hills - contd.

\begin{tabular}{|c|c|c|c|c|c|}
\hline S. No & Botanical name & Vernacular name & Family & Habit & Medicinal uses \\
\hline 85 & Dillenia pentagyna roxb. & Chinna kalinga & Dilleniaceae & Tree & Cooling agent for cattle \\
\hline 86 & Dioscoria pentaphylla L. & Injedigadda & Dioscoreaceae & Climber & Dysentery; Leprosy \\
\hline 87 & Diospyros ebenum J. Koenig. & Nalla uti & Ebenaceae & Tree & Astringent \\
\hline 88 & Diospyros melanoxylon Roxb. & Tumki & Ebenaceae & Tree & Dyspepsia; Diuretic \\
\hline 89 & Dodonea viscosa (L.) Jacq. Enum. & Bandaru & Sapindaceae & Shrub & Antipyretic; Bone fractures \\
\hline 90 & Eclipta prostrata (L.) L. & Gunta galijeru & Asteraceae & Herb & Asthma; Jaundice \\
\hline 91 & Echinops echinatus Roxb. & MullaBanthi & Asteraceae & Herb & Diuretic; Lice eradication \\
\hline 92 & Enicostema axillare. (Lam) Raynal & Gulividi & Gentianaceae & Herb & Scabies; Gout \\
\hline 93 & Entada pursaetha DC. & $\begin{array}{l}\text { Gila teega / Konda } \\
\text { chinta }\end{array}$ & Mimosaceae & Climber & Emetic \\
\hline 94 & Erythroxylum monogynum Roxb. & Devadari & Erythroxylaceae & Shrub & Stomachic; Diuretic \\
\hline 95 & Euphorbia hirta L. & Nanabala & Euphorbiaceae & Herb & Cough; Disentery \\
\hline 96 & Ficus benghalensis L. & Marri & Moraceae & Tree & Rheumatism \\
\hline 97 & Ficus microcarpa L.f. & Kondajuvvi & Moraceae & Tree & Wounds,Diabetes \\
\hline 98 & Gardenia gummifera.L.f. & Bikki & Rubiaceae & Tree & Ulcersl; Constipation \\
\hline 99 & Gardenia resinifera Roth. & Erribikki & Rubiaceae & Tree & Constipation; Bronchites \\
\hline 100 & Givotia moluccana (L.) Sreem & Tella poliki & Euphorbiaceae & Tree & Dandruf; Psoriasis \\
\hline 101 & Gmelia asiatica L. & Adavi Gummadi & Verbenaceae & Shrub & Dental Problems \\
\hline 102 & Gloriosa superba L.Sp.Pi. & $\begin{array}{l}\text { Nabhi / } \\
\text { Nagetigadda }\end{array}$ & Liliaceae & Climber & Leprocy; Abortifacient \\
\hline 103 & Glycosmis pentaphylla (Retz) DC. & Gonji & Rutaceae & Shrub & Diabetes; Eczema \\
\hline 104 & Grewia tiliifolia Vahl. & Adavichamanthi & Tiliaceae & & $\begin{array}{l}\text { Dysentery; Antidote to } \\
\text { opium poison }\end{array}$ \\
\hline 105 & $\begin{array}{l}\text { Guazuma tomentosa Lam. } \\
\text { Guazuma ulmifolia Lam. }\end{array}$ & Rudhracksha & Tiliaceae & Tree & Corpulence \\
\hline 106 & $\begin{array}{l}\text { Gymnema sylvestre (Retz) R.Br.ex } \\
\text { Sm }\end{array}$ & Podapatri & Asclepediaceae & Shrub & Diabetes \\
\hline 107 & Gyrocarpus asiaticus Willd. & Taniki /Nalla poliki & Hernandiaceae & Tree & Cancer \\
\hline 108 & Hardwickia binata Roxb. & Api & Fabaceae & Tree & Rheumatism \\
\hline 109 & Haldinia cordifolia (Roxb) Ridsdale & Rudra ganapa & Rubiaceae & Tree & Stomachic \\
\hline 110 & Hedtitus peberula (G.Don) Arn. & Chiruveru & Rubiaceae & Herb & Asthma; Bronchites \\
\hline 111 & Hedyotis corymbosa (L.) & Vermela - vemu & Rubiaceae & Herb & Diarrhoea; Stomachic \\
\hline 112 & Hedyotis herbacea L. & Chiriveru & Rubiaceae & Herb & Rheumatism; Febrifuge \\
\hline 113 & Heliotropium indicum L. & Nagadanthi & Boraginaceae & Herb & Ulcers; Eczema \\
\hline 114 & Helicters isora L. & Gooba thada & Sterculiaceae & Shrub & Diabetes; Dysentery \\
\hline 115 & $\begin{array}{l}\text { Hemidesmus indicus (L.) R.Br.ex } \\
\text { Schult. }\end{array}$ & Sugandhapala & Apocynaceae & Herb & $\begin{array}{l}\text { Cooling beverage; Cordio } \\
\text { tonic }\end{array}$ \\
\hline 116 & Hemionitis arifolia (Burm.f.) Moore & Rama bhanam & Hemionitidaceae & Herb & Antidiabetic \\
\hline 117 & Hugonia mystax L. & Kakibeera & Linaceae & Shrub & Antihelmenthic \\
\hline 118 & Hibiscus platanifolius (Willd.) & Kondagogu & Malvaceae & Tree & Diabetes; Rheumatism \\
\hline 119 & Hiptage benghalensis (L.) Kurz & Madhavi tega & Malphigiaceae & Climber & Diarrhoea; Dysentery \\
\hline 120 & $\begin{array}{l}\text { Holarrhena antidysenterica (L.) R. } \\
\text { Br. }\end{array}$ & $\begin{array}{l}\text { Kola musthi / pala / } \\
\text { kodisapala }\end{array}$ & Apocynaceae & Tree & Diarrohoea; Antidysentric \\
\hline 121 & Holostemma ada-kodein Schultes & $\begin{array}{l}\text { Tella jilledu / Peyyi } \\
\text { baddu }\end{array}$ & Asclepediaceae & Climber & Gonorrhoea; Diabetes \\
\hline 122 & $\begin{array}{l}\text { Hybanthus enneaspermus (L.) } \\
\text { Muell.Arg.Fragm. }\end{array}$ & Ratna purusha & Violaceae & Herb & Leucorrhoea; Diabetes \\
\hline 123 & Ichnocarpus frutescens (L.) R.Br. & Palateega & Apocynaceae & Climber & Blood purifier \\
\hline 124 & Indigofera aspalathoides Vahl.ex. & Sivavemu & Fabaceae & Herb & Oedema; Leprosy \\
\hline 125 & Ixora pavetta Andr. & Korivi/ Papidi & Rubiaceae & Tree & $\begin{array}{l}\text { Dysentery; Urinary } \\
\text { disorders }\end{array}$ \\
\hline
\end{tabular}


Table 1. Plants of Veligonda hills - contd.

\begin{tabular}{|c|c|c|c|c|c|}
\hline S. No & Botanical name & Vernacular name & Family & Habit & Medicinal uses \\
\hline 126 & Jasminum auriculatum Vahl. & Adavimalli & Oleaceae & Climber & Dropsy \\
\hline 127 & Justicia adhatoda L. & Addasaram & Acanthaceae & Shrub & Diabetes; Fever \\
\hline 128 & Lawsonia inermes $\mathrm{L}$. & Gorintaku & Lythraceae & Small Tree & Diarrhoea; Diabetes \\
\hline 129 & $\begin{array}{l}\text { Lannea coromandelica (Houtt.) } \\
\text { Merr. }\end{array}$ & Gumphena & Anacardiaceae & & Ulcers; dental diseases \\
\hline 130 & Lantana camara L. & Phallikampa & Verbanaceae & shrub & Tooth ache; Wounds \\
\hline 131 & Leonotis nepetiifolia (L) R.Br.Prodr & Ranabheri & Lamiaceae & Herb & Rheumatism \\
\hline 132 & Lepisanthes tetraphylla (Wall.) Radf. & Sali kunkudu & Sapindaceae & Tree & Skin diseases \\
\hline 133 & Leptadenia reticulate (Retz.) & Mukkupalateega & Asclepiadaceae & Climber & Abortifacient \\
\hline 134 & Limnophila indica (L.) Druce & Sambrani & Scrophulriaceae & Herb & Antiseptic; Dysentery \\
\hline 135 & Limonia acidissima Groff. & Velaga & Rutaceae & Tree & Stomachic; Astringent \\
\hline 136 & Listea glutinosa (Lour.) C.B.Rob. & Pulusumamidi & Lauraceae & Tree & Dysentery; Rheumatism \\
\hline 137 & Lygodium flexuosum (Linn.) & Mekasannu & Schizaeaceae & Climber & Scabies; Eczema \\
\hline 138 & Madhuca longifolia (Koen.) Macbr. & Ippa & Sapotaceae & Tree & Cough; Skin diseases \\
\hline 139 & $\begin{array}{l}\text { Mallotus philippensis (Lam.) Mull. } \\
\text { Arg. }\end{array}$ & Sinduri & Euphorbiaceae & Tree & Syphilis; Gonorrhoea \\
\hline 140 & Manikara hexandra (Roxb.) & Pala & Sapotaceae & Tree & Headache \\
\hline 141 & Mimusops elengi L. & Pogada & Sapotaceae & Tree & Diarrhoea \\
\hline 142 & Mimosa pudica (L.) & Aathipathi & Sapotaceae & Herb & Constipation; Leprosy \\
\hline 143 & Murraya paniculata (L.) Jack. & Naramusti & Rutaceae & Tree & Snake bite; dropsy \\
\hline 144 & Naravelia zeylanica (L.) DC & Korivi kattaku & Ranunkulaceae & Climber & Headache; Toothache \\
\hline 145 & Neptunia oleraceae Lour. & Neruthaluvapu & Fabaceae & Shrub & Syphilis \\
\hline 146 & Ochna obtusata DC. & Errijambi & Ochnaceae & shrub & Constipation; Asthma \\
\hline 147 & Olax scandens Roxb. & Mekabanda & Olacaceae & Climber & Anaemia; Fevers \\
\hline 148 & Opilia amentacea Roxb. & Nallamekabanda & Opilaceae & Climber & Hair tonic; Lice eradication \\
\hline 149 & Pavonia xylanica (L.) Cav. & $\begin{array}{l}\text { Adavi puttudu / } \\
\text { Chiru benda }\end{array}$ & Malvaceae & Herb & Blood motions \\
\hline 150 & Pentatropus capensis (L.f.) Bullock & Yedupullateega & Asclepiadaceae & Climber & Refrigerant \\
\hline 151 & Pergularia daemia (Forssk) Chiov. & Dushtapaku & Asclepiadaceae & Climber & Jaundice; Asthma \\
\hline 152 & Phoenix sylvestris (L.) Roxb. & Eetha & Arecaceae & Tree & Dysentery, Ulcers \\
\hline 153 & Phyllanthus amarus Schum \& Thonn & Nelausiri & Euphorbiaceae & Herb & Galactogogue; Jaundice \\
\hline 154 & Phyllanthus embllica L. & Nelli / Usiri & Euphorbiaceae & Tree & Febrifuge; Astringent \\
\hline 155 & Physalis minima L. & Budama & Solanaceae & Herb & Fever; Asthma \\
\hline 156 & Pimpinella tirupathensis $\mathrm{L}$. & Adavi kottimeera & Apiaceae & Herb & Ulcersl; Abortifacient \\
\hline 157 & Pithacalobium dulce (Roxb.) Benth. & Simachintha & Fabaceae & Tree & Leprosy; Diabetes \\
\hline 158 & Plumbago zylanica L. & Tella chitramulam & Plumbaginaceae & Herb & Scabies; Ulcers \\
\hline 159 & Pongamia pinnata (L.) Pierre & Kanuga & Fabaceae & Tree & Diabetes; Eczema \\
\hline 160 & Pouzolzia zeylanica (L.) Benn. & Uchchagadda & Urticaceae & Herb & Snake bite \\
\hline 161 & Premna tomentosa Willd & Narava/ Namari & Verbanaceae & Tree & Dropsy; Jaundice \\
\hline 162 & Pterocarpus marsupium roxb. & yegisa & Fabaceae & Tree & Cough; Skin Problems \\
\hline 163 & Pterocarpus santalilnus L.F. & Rakta chandanam & Fabaceae & Tree & Diabetes; Astringent \\
\hline 164 & Pterospermum xulocarpum (Gaertn.) & Tada & Sterculiaceae & Tree & Leucorrhoea \\
\hline 165 & Pueraria tuburosea Roxb.ex Willd. & $\begin{array}{l}\text { Chenchu gadda / } \\
\text { Bhoochakra }\end{array}$ & Fabaceae & Climber & Asthma; Rejuvinator \\
\hline 166 & $\begin{array}{l}\text { Rivea hypocrateriformis (Desr.) } \\
\text { Choisy }\end{array}$ & Boddi teega & Convolvulaceae & Shrub & Parturition \\
\hline 167 & Rhynchosia minima (L.) Dc. & Adavichikkudu & Fabaceae & Tree & Abortifacient \\
\hline 168 & Salvadora persica $\mathrm{L}$. & $\begin{array}{l}\text { Nalla uppili/ } \\
\text { Varagogu }\end{array}$ & Salvadoraceae & Tree & Asthma; Cough \\
\hline
\end{tabular}


Table 1. Plants of Veligonda hills - contd.

\begin{tabular}{|c|c|c|c|c|c|}
\hline S. No & Botanical name & Vernacular name & Family & Habit & Medicinal uses \\
\hline 169 & Santalum album $\mathrm{L}$. & $\begin{array}{l}\text { Chandanam, } \\
\text { Srigandham }\end{array}$ & Santalaceae & Tree & Diuretic; Skin eruptions \\
\hline 170 & Scilla hyacinthina (Roth) & Nakkeragadda & Liliaceae & Herb & Leprocy; diuretic \\
\hline 171 & Shorea roxburghii G.Don Gen.Syst & Jalari & Dipteerocarpaceae & Tree & Astringent; Rheumatism \\
\hline 172 & Shorea tumbeuggaia Roxb. & Tamba / Guggilam & Dipterocarpaceae & Tree & Ulcers \\
\hline 173 & $\begin{array}{l}\text { Solanum melongena L. var. insanum } \\
\text { L. }\end{array}$ & Chiruvanga & Solanaceae & Shrub & Hypertention; Diabetes \\
\hline 174 & Solanum surrattense Burm.F. & Errivanga & Solanaceae & Herb & $\begin{array}{l}\text { Helminthiasis; Tooth } \\
\text { Problems }\end{array}$ \\
\hline 175 & Solanum trilobatum L. & Mulla mushti & Solanaceae & Climber & Dyspepsia \\
\hline 176 & Soymida febrifuga (Roxb) A.Juss & Somi & Meliaceae & Tree & Diarrhoea; Dysentery \\
\hline 177 & Spondias pinnata (L.f.) Kurz & Adavimamidi & Anacardiaceae & & Astringent; Rheumatism \\
\hline 178 & Sphaeranthus indicus L. & Bodasaram & Asteraceae & Herb & $\begin{array}{l}\text { Aphrodisiac; } \\
\text { Anthehelmenthic }\end{array}$ \\
\hline 179 & Sterculia urens Roxb. & Thapasi & Sterculiaceae & Tree & Diabetes \\
\hline 180 & Strychnos calubrina L. & Nagamusti & Loganiaceae & Climber & Rheumatism; Diabetes \\
\hline 181 & Strychnos potatorum L.f. & Musthi & Loganiaceae & Tree & Kidney stones \\
\hline 182 & Strychnos potatorum L.F.Suppl. & Chilla & Loganiaceae & Tree & Stomachache \\
\hline 183 & Suregada angustifolia Baill. & Sapranchi & Euphorbiaceae & Shrub & Astringent \\
\hline 184 & Syzygium cumini (L.) Skeels & Neredu & Myrtaceae & Tree & Diarrhoea; Cough \\
\hline 185 & $\begin{array}{l}\text { Syzygium alternifolium (Wight) } \\
\text { Walp. }\end{array}$ & Mogi & Myrtaceae & Tree & Joint Paints \\
\hline 186 & Tamarindus indica $\mathrm{L}$. & Chinta & Fabaceae & Tree & Indigestion; Fever \\
\hline 187 & Tarenna asiatica $\mathrm{L}$. & Kommi & Rubiaceae & Shrub & Indigestion \\
\hline 188 & Terminalia arjuna (DC.) Wight\&Arn) & $\begin{array}{l}\text { Arjuna / Tella } \\
\text { maddi }\end{array}$ & Combretaceae & Tree & Blood motions \\
\hline 189 & Terminalia bellirica (Gaertn.) Roxb. & Thandra / tani & Combretaceae & Tree & Dysentery \\
\hline 190 & Terminalia pallida Brandis & Tella karaka & Combretaceae & Tree & Diarrhoea \\
\hline 191 & Terminallia chebula Retz. & Karaka & Combretaceae & Tree & Cough; Piles \\
\hline 192 & Thespisia populnia (L.) Correa & Gangaravi & Malvaceae & Tree & Boils; Ring Worms \\
\hline 193 & Tinospora cordifolia (Willd.) Miers & Tippa teega & Menispermaceae & Climber & Gonorrhea; Skin diseases \\
\hline 194 & Tribulus terrestris $\mathrm{L}$. & Palleru & Zygophyllaceae & Herb & Aphrodisiac; Leprocy \\
\hline 195 & Trichosanthes cucumeria L. & AdaviPotla & Cucurbitaceae & Climber & Diabetes \\
\hline 196 & Trichosanthes tricuspidata Lour. & Papara & Cucurbitaceae & Climber & Sores; Headache \\
\hline 197 & Tridax procumbens $\mathrm{L}$. & Gaddi chamanthi & Asteraceae & Herb & Dysentery; Wounds \\
\hline 198 & Triumfetta rhomboidea Jacq. & & Tiliaceae & Shrub & Ulcers; Leprocy \\
\hline 199 & Tylophora indica (Burm.f.) Merr. & Kakkupala & Asclepediaceae & climber & Asthma; Epilepsy \\
\hline 200 & Vanda spathulata L. & Nusti bhadhanika & Orchidaceae & Herb & Asthma; Consumption \\
\hline 201 & Vanda roxburghii Nicolson. & Veduru bhadhanika & Orchidaceae & Herb & Bone fracture \\
\hline 202 & Ventilago denticulata Willd. & Surati / Surudu & Rhamnaceae & Climber & Sprains; Malarial fever \\
\hline 203 & Vernonia anthelmintica (L.) & Adavi jeelakarra & Asteraceae & Herb & Digestion \\
\hline 204 & Vettiveria zizanioides (L.) Nash & Vattiveru & Poaceae & Herb & Diabetes; Cooling agent \\
\hline 205 & Viscum articulatum Burm.f. & Badanika & Viscaceae & Shrub & Ulcers; Febrifuge \\
\hline 206 & Vitex altissima L.f.syppl. & Nemaliadugu & Verbanaceae & Tree & Leprosy \\
\hline 207 & Walsura trifoliata (A.Juss) Harms & Valudu & Meliaceae & Tree & Emmenagogue \\
\hline 208 & Wattakaka volubilis (L.f.) Stapf & Kallisi & Asclepediaceae & Climber & Snake bite; Body pains \\
\hline 209 & Wrightia tinctoria (Roxb.) R.Br. & Reppala & Apocynaceae & Tree & Cough; Aphrodisiac \\
\hline 210 & Ziziphus mauritiana Lam. & Regu & Rhamnaceae & Tree & Scorpion sting; Diarrhoea \\
\hline 211 & Ziziphus xylopyrus (Retz.) Willd. & Gotti & Rhamnaceae & Tree & Asthma; Aphrodisiac \\
\hline
\end{tabular}




\section{Ethnology in Eastern Ghats of Andhra Pradesh}

The tribal people of Eastern Ghats of Andhra Pradesh originated long back of prehistoric era. The stone-age culture of these tribals is evidenced by micro and mega-lithic sites at upper Godavari (Sanapati and Sahu, 1966). Being Andhra Pradesh is drained by major rivers mainly in Eastern Ghats, the tribals also settled along the river streams which pass through the Ghats. These tribes have their own styles of culture, language, heritage, customs, religious practices, food habit etc., who mainly live deep in forests, unable to contact with civilized people. They mainly depend on minor forest produce, hunting and rarely on agriculture for their lively hood. Out of 33 types of tribals of Andhra Pradesh, 27 communities are confined to these isolated hills and adjacent plains.

\section{Yanadis}

The Yanadis are more primitive aboriginal and concentrated mainly in Chittoor, Kadapa, Nellore, Ongole and Guntur districts of Andhra Pradesh. Yanadis are the melanid Black Indians a hunter gather tribe (Rangha Rao, 1901) The tribes are set to be direct descendants of Paleolithic people. Chenchus and Yanadis both are from one parental stock and are believed to be originated in Nallamalai hill tracts (Raghavaiah, 1962). Yanadis speak only Telugu language with a characteristic dialect and accent. They don't have any special functions. Ceremonies or celebrations particular to them. They are integrated with Hindu social system and practice. The Yanadi lead a carefree, life with contentment and unbridled merriment. Their diet chiefly consists of vegetable food and animals, wild fowls and other birds of food value. They even dig rat holes and use them in menu. However, the best satiating food for them is fish. Honey gathering in forests and plains is also a common practice among them. Two sub-tribes are recognized with in Yanadis, based on their occupation, Manchi yanadi, the superior type and the Challa yanadis are inferior type and carry different names including Garapa Yanadis, Chettu yanadis, Kappa yanadis, based on their habitation and the food taken. The Yanadis are short statured with dark skin colour, platyrrhine nose, long head, prominent chin, thick lips and scanty hair both on head and body. They reside in huts usually construct adjacent to a water source. Yanadis living in and around forests keep themselves busy in collecting and selling minor products.

\section{Materials and Methods}

The study was conducted among the local tribes who were practising local medicine in near the villages of Veligonda hill ranges. Frequent field trips were carried out during the different seasons in June 2012 to June 2014. The data (Local name; Habit; Plant part used; Medicinal uses) were recorded through interviews among the traditional healers and local tribes in their language (Telugu). These medicinal plants were identified taxonomically with "Flora of Presidency of Madras" (Gamble, 1935). The collected specimens were deposited in the N. B. K. R. Medicinal Plant Research Institute, Vidya Nagar, Nellore.

\section{Results and Discussion}

The yanadis are the major tribes inhabiting in Veligonda hill ranges. They choose faith healing first. Traditional and herbal medicine next and modern medicine only when the first two are failed. They have not made any changes in their life style or tried to adapt to modernity.

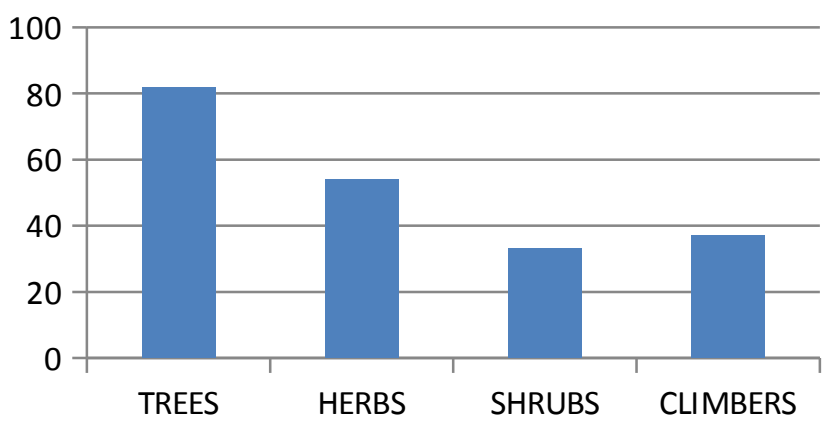

Fig. 1. Habit wise analysis of Veligonda hill range

Enumeration of 211 species belonging to 89 families used by local traditional healers was recorded in the Table 1. with Scientific Name; Vernacular Name; Family, Habit, Medicinal use. Out of 211 species maximum 82 species are trees (38.86\%); 54 (25.59\%) species are herbs; 36 (17.06) species are climbers and 34 (16.11) species are shrubs (Fig 1).

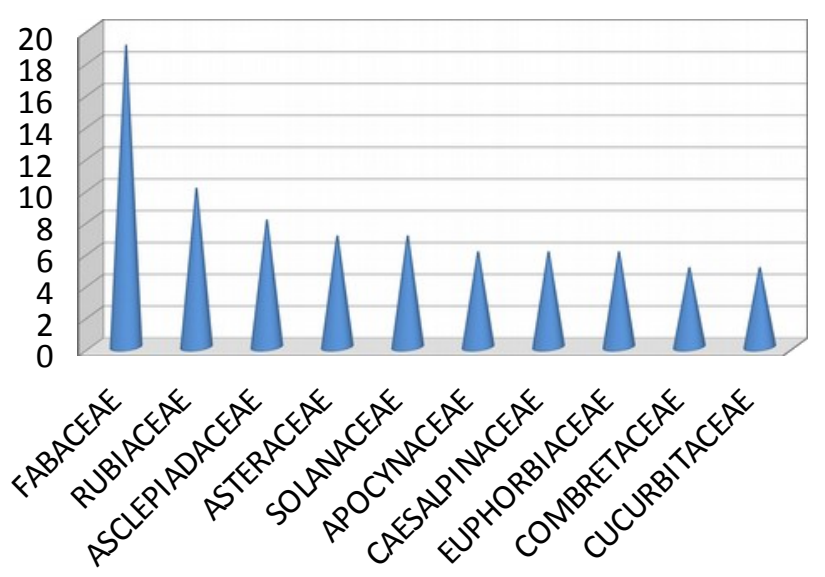

Fig. 2. Dominant families of Veligonda hill range

The rare and endangered plants like Red Sander and Sandal wood are the important 
species found these hills. The riparian vegetation is very rich in floristic value, consisting of Terminalia Species, Syzygium Species, wild mangoes (Anogeisus latifolia (Roxb ex. DC.) Wall. ex Guillem. \& Perr (Combretaceae), Hardwikia binata Roxb. (Caeslpiniaceae) etc. Dominant families are given in Fig 2.

\section{Conclusion}

Indian forests are the highest resources for medicinal plants. Due to over exploitation and various anthropogenic activities many medicinal plant species were become endangered. There is an urgent need to save this germ plasm for future generations. There are several plants with unknown medicinal value should be studied. Nearly 35000 tones of medicinal plants were being exported from India and Rs.3,500 crores are annually earning. So, there is an urgent need to develop conservation practices for sustainable utilization of medicinal plants.

\section{Competing Interest}

The authors declare that they have no competing interests.

\section{Authors' contributions}

SKMB and PSR designed and coordinated the study. PSR carried out field work, Data analysis, identification and manuscript preparation is done by SKMB.

\section{Acknowledgements}

The authors are thankful to the forest officials, tribals and local people for their help.

\section{References}

Andhra Pradesh State of Forest Report. 2013. Government of Andhra Pradesh.

Champion, H. G. and S. K. Seth. 1968. A Revised Survey of the Forest types of India. Government of India, New Delhi.

Gamble, J. S. 1915-36. Flora of Presidency of Madras. Adlard and Son Lrs Lonswn 1, 55.

Karve, Irawati. 1968. Hindu Society: An interpretation, $2^{\text {nd }}$ edn. Deshmuk prakashan, Poona.

Nair, M. P. and A. R. K, Sastry. 1998. Red Data Book of Indian Plants. BSI Publications.

Raghavaiah V, 1962. The Yanadis, New Delhi, India; Adimjati Sevak Sangh.

Ranga Rao, T. R. 1901. The Yanadis of Nellore district. Bull. Madras Govt. Museum IV (2): 87-113. 